\title{
Erratum to: Parameter identification and adaptive impulsive synchronization of uncertain complex-variable chaotic systems
}

\author{
Song Zheng
}

Published online: 25 December 2013

(C) Springer Science+Business Media Dordrecht 2013

\section{Erratum to: Nonlinear Dyn (2013) 74:957-967 DOI 10.1007/s11071-013-1015-4}

\section{Introduction}

In the original article [1], there are some errors in the proof of Theorem 1. Now, we point out the mistakes as follows:

The inequality (11) $\ln \alpha+\left(h-2 d^{*}\right)\left(t_{k}-t_{k-1}\right) \leq 0$, where $\alpha>1$ and $t_{k}-t_{k-1}>0$ in Theorem 1 implies that $h-2 d^{*}<0$. Therefore, with the Lyapunov type function (12)

$V(t)=e^{T}(t) \overline{e(t)}+\tilde{\Theta}^{T} \overline{\tilde{\Theta}}+\sum_{i=1}^{n} \frac{\left(d_{i}-d_{i}^{*}\right)^{2}}{k_{i}}$,

the inequality enlargement $D^{+} V(t) \leq\left(h-2 d^{*}\right) e^{T}$ $(t) \overline{e(t)} \leq\left(h-2 d^{*}\right) V(t)$ is incorrect, which appeared in the line 27 of the left column on page 960 in Ref. [1].

The online version of the original article can be found under doi:10.1007/s11071-013-1015-4.

\section{S. Zheng $(\bowtie)$}

Institute of Applied Mathematics, Zhejiang University

of Finance \& Economics, Hangzhou,

Zhejiang 310018, P.R. China

e-mail:songzheng070318@yahoo.com
For correcting the mistakes in the original paper, we slightly revise them and a correct version of Theorem 1 is given. The corresponding proof is also given in this paper.

\section{Correct version of Theorem 1 [1]}

Assumption 1 Suppose that there exists a constant diagonal matrix $H=\operatorname{diag}\left(h_{1}, h_{2}, \ldots, h_{n}\right)$ such that the complexvalued vector function $F(x, \Theta)$ satisfies

$$
\begin{aligned}
& (y-x)^{T} \overline{(F(y, \Theta)-F(x, \Theta))}+(F(y, \Theta) \\
& -F(x, \Theta))^{T} \overline{(y-x)} \leq(y-x)^{T} H \overline{(y-x)} .
\end{aligned}
$$

Theorem 1 Suppose Assumption 1 holds. The parameters updating law is designed as

$$
\begin{cases}\dot{\hat{\Theta}}=-\overline{g(y(t))}^{T} e(t), & t \neq t_{k}, \\ \Delta \tilde{\Theta}=A_{k} \tilde{\Theta}, & t=t_{k}, k=1,2, \ldots,\end{cases}
$$

where $\tilde{\Theta}=\hat{\Theta}-\Theta, \quad A_{k} \in R^{n \times n}$ is impulsive gain matrix. Let $\alpha_{k}=\lambda_{\max }\left(\left(I+A_{k}\right)^{T}\left(I+A_{k}\right)\right)<$ $1, \quad \beta_{k}=\lambda_{\max }\left(\left(I+B_{k}\right)^{T}\left(I+B_{k}\right)\right)<1, \mu_{k}=$ $\max \left(\alpha_{k}, \beta_{k}\right)$ and $d^{*}$ is the minimum value of the initial feedback strength $d_{i 0}\left(d_{i 0} \leq d_{i}, 1 \leq i \leq n\right)$. If there exist a constant $\alpha>1$ such that

$\ln \alpha \mu_{k}+\left(h-2 d^{*}\right)\left(t_{k}-t_{k-1}\right) \leq 0, \quad k=1,2, \ldots$ 
Then the response chaotic complex system (3) can synchronize the drive chaotic complex system (2) asymptotically with the impulsive controller (5), the adaptive controller (9) and the parameter update law (10) in Ref. [1]. Moreover, $\hat{\Theta} \rightarrow \Theta$, that is, all the unknown parameters are identified exactly.

Proof Select the following Lyapunov type function defined as

$$
V(t)=e^{T}(t) \overline{e(t)}+\tilde{\Theta}^{T} \overline{\tilde{\Theta}}
$$

For $t \neq t_{k}$, the derivative of (12) along the trajectories of (7) and Assumption 1, we have

$$
\begin{aligned}
D^{+} V(t)= & \dot{e}^{T}(t) \overline{e(t)}+e^{T}(t) \overline{\dot{e}(t)}+\dot{\tilde{\Theta}}^{T} \overline{\tilde{\Theta}}+\tilde{\Theta}^{T} \dot{\tilde{\tilde{\Theta}}} \\
= & {[F(y(t), \Theta)-F(x(t), \Theta)]^{T} \overline{e(t)} } \\
& +e^{T}(t) \overline{[F(y(t), \Theta)-F(x(t), \Theta)]} \\
& +[g(y(t)) \tilde{\Theta}]^{T} \overline{e(t)}+e^{T}(t) \overline{[g(y(t)) \tilde{\Theta}]} \\
& +\left[U_{1}\right]^{T} \overline{e(t)}+e^{T}(t) \overline{U_{1}} \\
& +\dot{\tilde{\Theta}}^{T} \overline{\tilde{\Theta}}+\tilde{\Theta}^{T} \dot{\overline{\tilde{\Theta}}} \\
\leq & e^{T}(t) H \overline{e(t)}+[g(y(t)) \tilde{\Theta}]^{T} \overline{e(t)} \\
& +e^{T}(t) \overline{[g(y(t)) \tilde{\Theta}]} \\
& +\dot{\tilde{\Theta}}^{T} \overline{\tilde{\Theta}}+\tilde{\Theta}^{T} \dot{\tilde{\tilde{\Theta}}}-2 \sum_{i=1}^{n} d_{i} e_{i}^{T}(t) \overline{e_{i}(t)}
\end{aligned}
$$

Denot $h=\max \left(h_{1}, h_{2}, \ldots, h_{n}\right)$ and $d^{*}=\min \left(d_{10}\right.$, $\left.d_{20}, \ldots, d_{n 0}\right)$, substitute Eqs. (9-10) into the above inequality, we further have

$$
\begin{aligned}
D^{+} V(t) & \leq\left(h-2 d^{*}\right) e^{T}(t) \overline{e(t)} \\
& \leq\left(h-2 d^{*}\right) V(t)
\end{aligned}
$$

with $h-2 d^{*}>0$.

This implies that

$$
\begin{aligned}
V(t) & \leq V\left(t_{k-1}^{+}\right) \exp \left(\left(h-2 d^{*}\right)\left(t-t_{k-1}\right)\right), \\
t & \in\left(t_{k-1}, t_{k}\right], \quad k=1,2, \ldots
\end{aligned}
$$

On the other hand, when $t=t_{k}$, from Eqs. (8) and (10) in Ref. [1], we have

$$
\begin{aligned}
& V\left(t_{k}^{+}\right) \leq e^{T}\left(t_{k}\right)\left(I+B_{k}\right)^{T}\left(I+B_{k}\right) \overline{e\left(t_{k}\right)} \\
& +\widetilde{\Theta\left(t_{k}\right)^{T}}\left(I+A_{k}\right)^{T}\left(I+A_{k}\right) \widetilde{\widetilde{\Theta\left(t_{k}\right)}} \\
& \leq \beta_{k} e^{T}\left(t_{k}\right) \overline{e\left(t_{k}\right)}+\alpha_{k} \widetilde{\Theta\left(t_{k}\right)} T \widetilde{\widetilde{\Theta\left(t_{k}\right)}} \\
& \leq \mu_{k} V\left(t_{k}\right)
\end{aligned}
$$

When $k=1$ in inequality (13), then for any $t \in\left(t_{0}, t_{1}\right]$ $V(t) \leq V\left(t_{0}^{+}\right) \exp \left(\left(h-2 d^{*}\right)\left(t-t_{0}\right)\right)$.

This leads to

$V\left(t_{1}\right) \leq V\left(t_{0}^{+}\right) \exp \left(\left(h-2 d^{*}\right)\left(t_{1}-t_{0}\right)\right)$.

Also from (14) we have

$$
\begin{aligned}
V\left(t_{1}^{+}\right) & \leq \mu_{1} V\left(t_{1}\right) \\
& \leq \mu_{1} V\left(t_{0}^{+}\right) \exp \left(\left(h-2 d^{*}\right)\left(t_{1}-t_{0}\right)\right)
\end{aligned}
$$

In the same way for $t \in\left(t_{1}, t_{2}\right]$, we have

$$
\begin{aligned}
V(t) & \leq V\left(t_{1}^{+}\right) \exp \left(\left(h-2 d^{*}\right)\left(t-t_{1}\right)\right) \\
& \leq \mu_{1} V\left(t_{0}^{+}\right) \exp \left(\left(h-2 d^{*}\right)\left(t-t_{0}\right)\right) .
\end{aligned}
$$

In general for any $t \in\left(t_{k}, t_{k+1}\right]$, one finds that

$$
V(t) \leq \mu_{1} \mu_{2} \ldots \mu_{k} V\left(t_{0}^{+}\right) \exp \left(\left(h-2 d^{*}\right)\left(t-t_{0}\right)\right)
$$

From the condition given in the Theorem 1, we have $\mu_{k} \exp \left(\left(h-2 d^{*}\right)\left(t_{k+1}-t_{k}\right)\right) \leq \frac{1}{\alpha}, k=1,2, \cdots$.

Notice that

$$
\begin{aligned}
t-t_{0}= & \left(t-t_{k}\right)+\left(t_{k}-t_{k-1}\right)+\left(t_{k-1}-t_{k-2}\right) \\
& +\cdots+\left(t_{2}-t_{1}\right)+\left(t_{1}-t_{0}\right) .
\end{aligned}
$$

Thus, the inequality(15) can be further rewriten as

$$
\begin{aligned}
V(t) \leq & \mu_{1} \mu_{2} \ldots \mu_{k} V\left(t_{0}^{+}\right) \exp \left(\left(h-2 d^{*}\right)\left(t-t_{0}\right)\right) \\
= & V\left(t_{0}^{+}\right)\left[\mu_{1} \exp \left(\left(h-2 d^{*}\right)\left(t_{1}-t_{0}\right)\right)\right] \times \cdots \\
& \times\left[\mu_{k} \exp \left(\left(h-2 d^{*}\right)\left(t_{k}-t_{k-1}\right)\right)\right] \\
& \quad \exp \left(\left(h-2 d^{*}\right)\left(t-t_{k}\right)\right) \\
\leq & V\left(t_{0}^{+}\right) \frac{1}{\alpha^{k}} \exp \left(\left(h-2 d^{*}\right)\left(t-t_{k}\right)\right)
\end{aligned}
$$

Therefore, $V(t) \rightarrow 0$ when $k \rightarrow \infty$ because of $\alpha>1$, which implies that all the errors $e(t) \rightarrow 0$. So the complete synchronization between the controlled chaotic complex system (3) and (2) in [1] is realized.The proof is completed.

Remark 1 From proof of Theorem 1, we know that the synchronization error will converge to zero. Fom parameters update laws (10), we find that $\dot{\hat{\Theta}}=0$ when $e(t)=0$, which implies that $\hat{\Theta}$ approach to some constants and this does not ensure that $\hat{\Theta} \rightarrow \Theta$. To identify the unknown parameters, we suppose that the nonlinear vector function $g^{T}(y(t))$ should be linearly independent on the synchronization manifold $y=x$. According to the results of Ref. [2], then the unknown parameters $\Theta$ can be identified by $\hat{\Theta}$ as $k \rightarrow \infty$. 


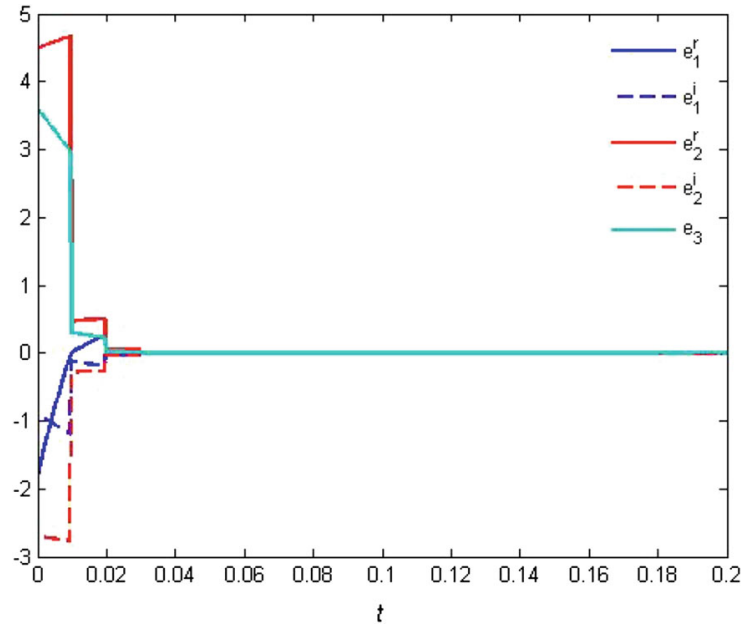

Fig. 1 Errors of real and imaginary parts of $e_{i}(t)$.

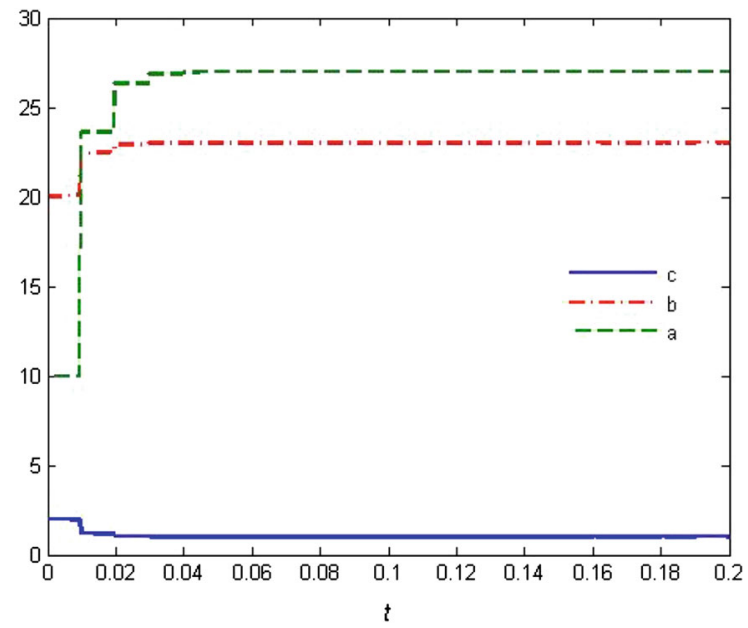

Fig. 2 Evolution of the three parameters' estimation with time $t$.

In the numerical simulation, let $d_{i 0}=20, \alpha=$ $2, A_{k}=\operatorname{diag}(-0.8,-0.8,-0.8)$ and $B_{k}=\operatorname{diag}(-0.5$, $-0.5,-0.5)$, then, $\mu_{k}=0.25$ and $h=79$ in [1]. From Eq. (11), we can obtain $0<t_{k}-t_{k-1} \leq-\frac{\ln \alpha \mu_{k}}{h-2 d^{*}}=$ 0.0178 , thus, let the impulsive interval $t_{k}-t_{k-1}=0.01$. Figures 1 and 2, respectively, show the synchronization errors $e_{i}(t)$ and the identified parameters under the updating laws (10).
Acknowledgments The authors are grateful to Prof. Wei Shao for recognizing this error and his very useful comments. This work was jointly supported by the National Science Foundation of China (Grant Nos. 11102076 and 11202085), the Natural Science Foundation of Zhejiang Province (Grant No. LY13F030016) and the Foundation of Zhejiang Provincial Education Department (Grant No. Y201328316).

\section{References}

1. Zheng, S.: Parameter identification and adaptive impulsive synchronization of uncertain complex-variable chaotic systems. Nonlinear Dyn 74, 957-967 (2013)

2. Yu, W., Chen, G., Cao, J., Lu, J., Parlitz, U.: Parameter identification of dynamical systems from time series. Phys. Rev. E 75, 067201 (2007) 